\title{
Comparison Of Tolerant And Susceptible Cultivars Revealed The Roles of Circular RNAs In Rice Responding To Salt Stress
}

Junliang Yin ( $\nabla$ w.yinzi@163.com )

Yangtze University https://orcid.org/0000-0002-3375-8622

Yike Liu

Hubei Academy of Agricultural Sciences

Lin Lu

Northwest A\&F University

Jian Zhang

Yangtze University

Shaoyu Chen

Hubei Academy of Agricultural Sciences

\section{Baotong Wang}

Northwest A\&F University

\section{Research Article}

Keywords: circRNA, Oryza sativa L., salt stress, circRNA-miRNA-mRNA network

Posted Date: June 11th, 2021

DOl: https://doi.org/10.21203/rs.3.rs-550126/v1

License: (9) (i) This work is licensed under a Creative Commons Attribution 4.0 International License.

Read Full License

Version of Record: A version of this preprint was published at Plant Growth Regulation on November 15th, 2021. See the published version at https://doi.org/10.1007/s10725-021-00772-y. 


\section{Abstract}

As a newly characterized class of noncoding RNAs, circular RNAs (circRNAs) have been identified in many plant species, and play important roles in plant stress responses. However, little is known about how salt stress mediates the expression of circRNAs in rice. In this study, we identified circRNAs from root tissues of salt-susceptible recipient cultivar 93-11 and salt-tolerant introgression line 9L136. A total of 190 circRNAs were identified. Among them, 93 circRNAs were differentially expressed under salt stress in 93-11 (36 up- and 57 down-regulated) and 95 in 9L136 (46 up- and 49 down-regulated). Salt stress significantly decreased the average expression level of circRNAs in 93-11, but circRNA expression levels were slightly increased in 9L136, suggesting that circRNAs had different response patterns in these two cultivars. Function annotation and enrichment analysis indicated that, through cis-regulation and circRNA-miRNA-mRNA network regulation, those induced circRNAs were commonly involved in transcription, signal transduction, ion transportation, and secondary metabolism. Compared to 93-11, salt-induced circRNAs in line 9L136 targeted more stress response genes participating in transcription regulation, ion transportation, and signal transduction, which may contribute to the salt tolerance of 9L136. Summarily, this study revealed the common response of rice circRNAs to salt stress, and the possible circRNA-related salt tolerance mechanisms of 9L136.

\section{Introduction}

During the past decade, a novel type of noncoding RNAs, termed as circular RNAs (circRNAs), have been discovered and widely studied (Zhou et al, 2018). Unlike formerly known noncoding RNAs, circRNAs are covalently closed RNA circles formed by head-to-tail splicing of transcripts that are devoid of the 5'-cap and 3'-tail (Zhu et al, 2019a). With the development of high-throughput sequencing technologies, numerous circRNAs have been discovered in both animals and plants in recent years (Zhao et al, 2019). In general, circRNAs can be classified into three main categories based on their genomic location, exonic circRNAs, intronic circRNAs, and intergenic circRNAs ( $\mathrm{Li}$ et al, 2019). Some circRNAs are conserved, more abundant than their linear counterparts, and exhibit cell, and tissue-specific expression patterns in different plant species, suggesting the potential physiological functions of these circRNAs (You et al, 2015; Zhao et al, 2017).

Although circRNAs are generally expressed at low levels, they may be involved in many physiological and molecular processes. Several circular RNAs were recently demonstrated to possess microRNA (miRNA) binding sites, which may function as sponges to sequester miRNAs and prevent their interactions with target mRNAs (Yin et al, 2017; Zhou et al, 2018). However, the miRNA sponge functions of most circRNAs have not been proved experimentally. In addition, many studies have proposed that some circRNAs could be translated into proteins in a cap-independent manner. For example, Zhang et al, (2018) showed that a circRNA containing an open reading frame (ORF) driven by the internal ribosome entry site (IRES) can translate a functional amino acid protein termed as SHPRH-146aa. Moreover, circRNAs can interact with functional proteins to exert their functions in multiple processes. Li et al, (2017) reported that in humans, double-stranded RNA-binding domains containing immune factors NF90 and NF110 were components of 
circRNPs in the cytoplasm and could enhance circRNA production in the nucleus through stabilizing intronic RNA pairs. However, the general functions of most circRNAs remain far from clear.

In plants, circRNAs have been identified and studied in increasing number of species, including Arabidopsis (Liu et al, 2017), rice (Ye et al, 2016), wheat (Wang et al, 2017a), tomato (Tan et al, 2017; Yin et al, 2018), kiwifruit (Wang et al, 2017b), soybean (Zhao et al, 2017), potato (Zhou et al, 2018), tea (Tong et al, 2018) and cucumber (He et al, 2020; Zhu et al, 2019a). Similar to animal circRNAs, plant circRNAs are abundant, conserved, and expressed at low concentrations and in a tissue-specific manner. However, plant circRNAs also have distinct features, such as a significantly lower proportion of both repetitive elements and reverse complementary sequences in introns flanking exonic circRNAs (Meng et al, 2016; Chu et al, 2020). Moreover, plant circRNAs might also be involved in a broad range of biological pathways (Chen et al, 2018). For example, some circRNAs exhibited different expression patterns during fruit ripening (Yin et al, 2017) and under biotic and abiotic stresses (e.g. dehydration, salt, and heat stresses) (Wang et al, 2017a; Wang et al, 2017b; Pan et al, 2018; Zhu et al, 2019a). In addition, circRNAs can affect their parental genes in cis- or trans-actions. In rice, circRNAs has been found to negatively regulate the expression of their parental genes (Lu et al, 2015). In Arabidopsis, Conn et al, (2017) demonstrated that certain circRNAs have the ability to skew splicing preference and increase abundance of their cognate exon-skipped alternative splicing (AS) mRNA variants, thereby revealing that specific circRNAs have the ability to regulate the splicing of their cognate AS mRNAs. Several studies in animals have shown that circRNAs could serve as "miRNA sponges" to regulate mRNA expression, but "miRNA sponges" function of circRNAs in plants have not been experimentally characterized. These findings suggest that biological roles of circRNAs in plants are diverse. However, to date, few experiments have analyzed the functional and formation mechanisms of circRNAs in plants.

Globally, rice (Oryza sativa L.) is one of the most important crops and is quite sensitive to salinity (Gong et al, 2006). In rice, Ye et al, (2015) conducted a genome-wide identification of circRNA for the first time, and revealed the potential regulatory functions of 27 differentially expressed exonic circRNAs in response to Pi-starvation stress. Furthermore, Lu et al, (2015) identified 2,354 circRNAs through deep sequencing, and the computational analysis of transcriptome data and transgenic analysis suggested that circRNA and its linear form could reduce the expression of its parental gene. Ye et al, (2016) identified nearly 3,000 circRNAs in rice and found that non-GT/AG splicing signals were common in rice circRNAs. However, to our knowledge, there is still limited data on the role of circRNAs in the salt stress resistance of rice (Wang et al. 2019). In this study, we aimed to provide insight into the possible roles of circRNAs in response to salt stress, which could provide a basis for further functional studies of rice circRNAs.

\section{Materials And Methods}

\section{CircRNA identification}

Rice RNA-seq dataset used in this study was accessible in NCBI (National Center for Biotechnology Information) under the SRA (Sequence Read Archive) database (BioProject PRJNA395311). The dataset 
includes the control $(0 \mathrm{~h}, \mathrm{CK})$ and salt treatment $(1 \mathrm{~h}$ after treatment, $\mathrm{NaCl})$ samples from roots of the recurrent parent 93-11 and introgression line 9L136. Different samples were sequenced in three biological replicates (Wang et al, 2017c). Low quality reads, including (1) unknown $(\mathrm{N})$ bases that were greater than $5 \%$, (2) contained adaptor sequences, and (3) contained more than $50 \%$ bases with $Q \leq 20$, were removed. The remaining high-quality reads were mapped to the previously reported Oryza sativa $\mathrm{L}$. reference genome RGAPv7 (MSU Rice Genome Annotation Project Release 7) using BWA (v0.7.15, mem T 19) (Zhu et al, 2019a). CIRI2 (v2.05) is considered to be the most reliable software, with remarkably balanced sensitivity and reliability (Gao et al, 2017), thus the output SAM files of BWA were inspected using CIRI2 to identify circRNAs. CircRNA expression levels were normalized to be SRPBM (SRPBM = number of circular reads/number of mapped reads (units in billion)/read length). Those known rice circRNAs were download from the database PlantCircBase (Chu et al. 2017). Junction reads were manually extracted and blastn was performed against known rice circRNAs (-evalue 0.05).

\section{CircRNA annotation and $\mathrm{GO}$ enrichment}

Databases including $\mathrm{Nr}$ (NCBI non-redundant protein sequences), GO (Gene Ontology), KEGG (Kyoto Encyclopedia of Genes and Genomes), KOG/COG (Clusters of Orthologous Groups of roteins), Swiss-Prot (A manually annotated and reviewed protein sequence database, https://www.uniprot.org/), and Pfam (Protein family) were used to perform gene function annotation (Yin et al, 2018). The identification of differentially expressed circRNAs was performed using the R package EBSeq. The cut-off criteria were set as an SRPBM value large than 0.5 and a fold change large than 2. GO annotations of the parent genes of differentially expressed circRNAs were collected and used to perform GO enrichment using topGO with default parameters. Venn diagram was draw using online tools

(http://bioinformatics.psb.ugent.be/webtools/Venn/). Circos and Violin diagrams were draw using correspondence $\mathrm{R}$ packages.

\section{Prediction of circRNA-miRNA-mRNA relationships}

To construct the circRNA-miRNA-mRNA network, we collected the mature sequences of rice miRNAs from the miRBase. Then the miRNAs sponged by circRNAs were predicted by TargetFinder using default parameters (Zhu et al, 2019a). Meanwhile, rice coding genes (mRNAs) targeted by collected miRNAs were predicted by searching the CDS sequences of the RGAPV7 reference genome with an online tool (http://plantgrn.noble.org/psRNATarget/) (Fang et al, 2020). The circRNA-miRNA-mRNA regulating network was generated by Cytoscape (v3.6.0) (Yin et al, 2018). The eggNOG class annotations of these miRNAs targeted mRNAs were collected and used to interpret the possible regulating roles of circRNAs.

\section{Validation of circRNAs}

To further confirm the circRNAs identified from rice, total RNA was extracted using the Plant RNA Kit (Omega, London, UK). The cDNA was then synthesized from $500 \mathrm{ng}$ of total RNA using the RevertAid First Strand cDNA Synthesis Kit (Thermo Scientific, USA) according to the manufacturer's protocol. The 
divergent PCR primers were designed using the "out-facing" strategy to exclude linear mRNA from amplification. Sanger sequencing was further used to confirm the presence of back-splicing junctions.

\section{Results}

\section{Identification of rice circRNAs}

To explore candidate circRNAs related to salt tolerance, the RNA-seq dataset obtained from the root tissues of salt-susceptible recipient cultivar 93-11 and salt-tolerant introgression line 9L136 (seedlings after $0(\mathrm{CK})$ and $1 \mathrm{~h}$ of $\mathrm{NaCl}$ treatment $(\mathrm{Na})$ ) was used to perform the identification, expression profiling, and function annotation of rice circRNAs. In total, 864 million reads were obtained from 12 libraries, and about $94.98 \%$ of the reads could be mapped to the reference genome by BWA (Table 1). Then, the mapping SAM files were scanned by the CIRI2 tool with the default parameters, resulting in the identification of 14,261 junction reads (Fig. 1A). Finally, 190 circRNAs supported by at least two unique back-spliced reads were detected, with 118 in 93-11 and 130 in 9L136 (Fig. 1B). Among these 190 circRNAs, 83 and 66 were detected from CK and $\mathrm{Na}$ in 93-11, respectively; meanwhile, 88 and 82 were found from CK and $\mathrm{Na}$ in 9L136, respectively. Moreover, 22 circRNAs were detected in all samples (Fig. $1 B)$. Further validation revealed that the majority of junction reads $(12,801,90 \%)$ overlapped with the reported circRNAs, hence suggesting the reliability of detected circRNAs in this study.

Table 1. Genome-wide identification of circRNAs in recipient 93-11 and introgression line 9L136.

\begin{tabular}{|lllll|}
\hline Sample & Total reads & Mapped reads & Junction reads & circRNA \\
\hline 93-11_CK1 & 57072607 & $54478269(95.45 \%)$ & 593 & 42 \\
\hline 93-11_CK2 & 53398323 & $51245137(95.97 \%)$ & 680 & 42 \\
\hline 93-11_CK3 & 78402777 & $74639808(95.20 \%)$ & 874 & 41 \\
\hline 93-11_Na1 & 60775605 & $58516223(96.28 \%)$ & 1240 & 40 \\
\hline 93-11_Na2 & 67457536 & $65887625(97.67 \%)$ & 1802 & 35 \\
\hline 93-11_Na3 & 54053559 & $52701664(97.50 \%)$ & 956 & 28 \\
\hline 9L136_CK1 & 68664588 & $66099177(96.26 \%)$ & 625 & 47 \\
\hline 9L136_CK2 & 61652454 & $59158113(95.95 \%)$ & 594 & 39 \\
\hline 9L136_CK3 & 70258564 & $66018921(93.97 \%)$ & 1072 & 45 \\
\hline 9L136_Na1 & 106475882 & $101152879(95.00 \%)$ & 2527 & 47 \\
\hline 9L136_Na2 & 84584242 & $82262283(97.25 \%)$ & 1683 & 39 \\
\hline 9L136_Na3 & 101699931 & $88931303(87.44 \%)$ & 1615 & 36 \\
\hline Total & 864496068 & $821091402(94.98 \%)$ & 14261 & 190 \\
\hline
\end{tabular}




\section{Characteristics of rice circRNAs}

Genomic origin analysis showed that the largest majority $(129,67.89 \%)$ of the 190 identified circRNAs were intergenic circRNAs, 47 (24.74\%) were exonic circRNAs, and the remaining 14 (7.34\%) were intronic circRNAs (Fig. 2A). Genomic original location analysis suggested these circRNAs were widely scattered on all chromosomes (Fig. 2B). However, unlike coding genes, genomic density analysis showed that circRNA genes were unevenly distributed and more commonly found at both ends of chromosomes. More circRNAs (26 and 26) were transcribed from Chr1 (chromosomes 1 ) and Chr4 respectively, followed by Chr6 (22) and Chr2 (22), both of which had 22 (Fig. 2D). Further analysis revealed that the number of circRNAs was significantly correlated with chromosome length (Fig. 2E, $\left.R^{2}=0.6759 * \star \star\right)$. Alternative circularization analysis identified 50 alternative back-splicing circularization events originating from 19 unique chromosome loci. In total, 11 of 19 loci produced two different circRNA isoforms, five produced three distinct isoforms, two produced four isoforms, and one produced five isoforms (Fig. 2C, Supplementary table 1).

\section{Expression patterns of rice circRNAs under salt stress}

To study the effect of salt stress on rice circRNAs expression, we compared the average expression levels of all detected circular RNAs. A Wilcoxon rank-sum test indicated that, generally, the average expression levels of circRNAs were significantly decreased by salt stress in recipient 93-11 ( $p$-value $<0.05$. Fig. 3A), whereas the expression levels of circRNAs were slightly increased by salt stress in introgression line 9L136. In order to reveal rice circRNAs that may have biological functions in response to salt stress, we compared the expression profiles of differentially expressed circRNAs between control and salt stress samples. Among the 190 circRNAs, 96 circRNAs were found to be significantly differentially expressed under salt stress, including 93 in recipient 93-11 (36 up- and 57 down-regulated, Supplementary table 2) and 95 in introgression line 9L136 (46 up- and 49 down-regulated, Supplementary table 3) (Fig. 3B).

\section{Functional categorization of stress-regulated parent genes}

CircRNAs have been proposed to regulate their parent coding genes (Lu et al, 2015; Cheng et al, 2018); thus, we predicted and annotated the parent genes of these differentially expressed circRNAs. GO enrichment analysis of the parent genes of these differentially expressed circRNAs showed that, in salt tolerance introgression line 9L136, GO terms related to: (1) Biological Process (BP), including transport (GO:0006810), establishment of localization (GO:0051234), translation (G0:0006412), and localization (GO:0051179); (2) Cellular Component (CC), including cytosol (GO:0005829) and protein complex (GO:0043234); (3) Molecular Function (MF), including nucleoside-triphosphatase activity (G0:0017111), pyrophosphatase activity (G0:0016462), hydrolase activity, acting on acid anhydrides (GO:0016817), were specifically enriched (Fig. 4A, Supplementary table 2). In salt-susceptible recipient cultivar 93-11, GO terms related to the generation of precursor metabolites and energy (G0:0006091) of $\mathrm{BP}$, as well as electron carrier activity (G0:0009055) and actin binding (G0:0003779) of MF, were specifically enriched (Fig. 4B, Supplementary table 3). 


\section{Prediction of the circRNA-miRNA-mRNA regulating network}

In addition to regulating parent genes, circRNAs were found to competitively and specifically bind miRNAs to prohibit miRNAs from the epigenetic regulating of target mRNAs (Liu et al, 2017). In the present study, 27 miRNAs were predicted to be sponged by 19 differentially expressed circRNAs in 9L136, and 37 miRNAs were predicted to be sponged by 26 differentially expressed circRNAs in 93-11, suggesting that these circRNAs may function as miRNA sponges in rice (Supplementary tables 4-7). Furthermore, 426 mRNAs were predicted to be the targets of 27 miRNAs in 9L136, and 327 mRNA were predicted to be the targets of 37 miRNAs in 93-11 (Supplementary tables 4-7). EggNOG class annotation analysis suggested that 19 circRNAs of 9L136 play roles in the regulation of physiological and biochemical processes, including transcription, signal transduction, translation, secondary metabolism, and inorganic ion transport, etc (Fig. 5A, Supplementary figure 1, Supplementary table 5). Similar results were found in 93-11. CircRNAs also play roles through involving EggNOG classes such as transcription, translation, signal transduction, and secondary metabolism (Fig. 5B, Supplemental figure 1, Supplementary table 7).

\section{Experiment confirmation of salt-induced circRNA in rice}

To further confirm the existence of rice circRNAs detected by this study, divergent primers were designed to perform the divergent polymerase chain reaction (PCR). Ten selected circRNAs were confirmed to be processed from back-splicing, demonstrating the reliability of our cicRNA identification based on highthroughput sequencing (Fig. 6. Supplementary table 8).

\section{Discussion}

\section{CircRNA identification}

Rice circRNAs had been identified in multiple tissues, including leaves, anthers, pistils, seeds, shoots, and roots, implying the roles of circRNAs in different rice growth and development stages (Lu et al, 2015; Ye et al, 2015; Chu et al, 2017; Ye et al, 2017). However, it is still much unclear how circRNAs participate in the response of rice to salt stress. Previously, Wang et al, (2017c) developed salt-tolerant introgression line 9L136 using wild Oryza rufipogon accession as the donor, and Oryza sativa indica cultivar 93-11 as the recipient. RNA-seq profiling revealed more salt stress-induced differentially expressed genes $(1,391)$ in introgression line 9L136 than recipient 93-11. In this study, this RNA-seq profiling dataset was used to explore candidate salt tolerance-related circRNAs. In total, 190 circRNAs supported by at least two unique back-spliced reads were detected, including 118 in 93-11 and 130 in 9L136 (Fig. 1B).

\section{circRNAs related to salt stress}

In plants, many circRNAs have been reported to exhibit stress-inducible expression patterns (Zhao et al, 2019). For example, Zhu et al, (2019) identified 2,787 circRNAs in cucumber, with 1,934 in root and 44 in leaf being differentially regulated under salt stress. Annotation and enrichment analysis of both parental 
genes and target mRNAs of salt-induced circRNAs suggested that circRNAs may paly roles in salt stress response by regulating abiotic stress associated genes. In Arabidopsis, Pan et al, (2018) uncovered 1,583 heat-specific circRNAs, suggesting these circRNAs may participate in heat stress response. Plant salt stress tolerance is a very complex regulating progress involving in signal transduction, substance and energy metabolism (Zhu et al, 2019b, c). In this study, Wilcoxon rank-sum test indicated that salt stress significantly decreased the average expression level of circRNAs in salt-susceptible cultivar 93-11 ( $p$-value $<0.05$. Fig. 3A), but slightly increased it in salt tolerant introgression line 9L136.

In order to reveal rice circRNAs that may have biological functions in response to salt stress, we compared the expression profiles of differentially expressed circRNAs between control and salt stress samples. Among the 190 circRNAs, 96 circRNAs were significantly differentially induced by salt stress, including 93 in recipient parent 93-11 (36 up- and 57 down-regulated, Supplementary table 2) and 95 in introgression line 9L136 (46 up- and 49 down-regulated, Supplementary table 3) (Fig. 3B). Since circRNAs have been proposed to be positive or negative regulators on their parent coding genes ( $L u$ et al, 2015; Cheng et al, 2018), we predicted and annotated the parent genes of these differentially expressed circRNAs. GO enrichment analysis implied that the parent genes of differentially expressed circRNAs in 9L136 were involved in more biological and biochemical processes than in salt-susceptible cultivar 93-11 (Fig. 4A, Supplementary table 2; Fig. 4B, Supplementary table 3). Considering the fact that 9L136 is much more tolerant to salt stress than 93-11, it seems that circRNAs contributed to the salt-tolerant phenotype in introgression line $9 \mathrm{~L} 136$.

\section{CircRNA-miRNA-mRNA regulating network prediction}

Besides regulating parent genes, previous studies suggested that circRNAs could bind specific miRNAs to repress the regulating ability of miRNAs (Liu et al, 2017). In this study, 27 miRNAs and 37 miRNAs were predicted to be competitively bound by 19 and 26 differentially expressed circRNAs in 9L136 and 93-11, respectively (Supplementary tables S4-7). Among those miRNAs, some were reported to be closely related to plant stress responses in rice. For example, osa-mir2925 was reported to be up-regulated by salt stress in rice, and osa-mir2925 potentially targets stress-response-associated genes, such as LOC_Os05g24780 (calcium-binding protein CML21), LOC_Os09g28200 (Heat stress transcription factor B-4c) in 9L136, and LOC_Os01g43320 (GABA transporter) in 93-11 (Fig. 5) (Wang et al, 2017c). Another osa-miR2925 was competitively bound by circular RNA Chr10:18818949|18958248 in 9L136 and by Chr6:2046190|2059120 in 93-11. Interestingly, Chr10:18818949|18958248 and Chr6:2046190|2059120 showed distinct regulation patterns in 9L136 and 93-11 under salt stress. In salt-tolerant line 9L136, Chr10:18818949|18958248 was down-regulated, which may decrease its binding effect to osa-mir2925. Meanwhile, in 93-11, Chr6:2046190|2059120 was up-regulated, which may promote the sponge effect of Chr6:2046190|2059120 to osa-mir2925. It seems that in this regulation network, the distinct regulation patterns of circular RNAs in salt-resistant line 9L136 and salt-susceptible cultivar 93-11 result in the opposite regulating effect on miRNA, which further leads to the differential expression of stress-responseassociated mRNAs, and may contribute to the salt-resistant phenotype of $9 \mathrm{~L} 136$ and salt-susceptible phenotype of 93-11. 
Additionally, it should be noticed that several miRNAs competitively bound by circRNAs were markedly different between the two rice cultivars, thus indicating the different regulating networks of circRNAs in the two rice cultivars in response to salt stress (Fig. 5. Supplementary tables 4-7). For example, the salttolerant introgression line 9L136 showed several unique regulation networks. In 9L136, osa-miR2102-5p and osa-mir5809 were predicted to be competitively bound by circRNAs Chr10:18818949|18958248 and Chr7:14201482|14303571, respectively. The osa-miR2102-5p has been widely reported to be involved in salt and drought stresses in Gramineae plants, such as barley (Zare et al, 2019), maize (Wang et al, 2014), and Spartina alterniflora (Qin et al, 2015). In rice, osa-mir5809 was reported to be involved in salt (Huang et al, 2019) and heat stresses (Mangrauthia et al, 2017), as well as leaf senescence (Xu et al, 2014). Both circRNA Chr10:18818949|18958248 and Chr7:14201482|14303571 were down-regulated by salt stress in 9L136, which could decrease the competitive binding of circRNAs to miRNA osa-miR2102$5 p$, and further enhance the suppression of osa-miR2102-5p to target mRNAs. These findings implied the involvement of circRNAs in the response of rice to salt stress. CircRNAs may also have various biological functions during the growth and stress responses of rice. However, the function of circRNAs in rice needs further experimental validation. Further analysis demonstrated that 426 mRNAs were predicted to be the targets of 27 miRNAs in 9L136, and 327 mRNA were predicted to be the targets of 37 miRNAs in 93-11 (Supplementary tables 4-7). EggNOG class annotation analysis revealed that these mRNAs participated in the regulation of physiological and biochemical processes, including transcription, signal transduction, and secondary metabolism (Fig. 5, Supplementary figure 1, Supplementary tables 4-7). Among those miRNA targets, more mRNAs were differentially expressed in 9L136 (108) than in 93-11 (83), suggesting a more complex regulation network in the salt stress tolerance introgression line 9L136.

\section{Expression patterns of circRNAs and their corresponding parental genes in different cultivars}

Previous studies in several species have suggested that most circRNAs regulate the expression level of their corresponding parental genes. In rice, Lu et al, (2015) proposed that circRNA and its linear form may act as a negative regulator of its parental gene. However, expression profiles of circRNAs in tea and Arabidopsis showed a positive correlation between circRNAs and their parental genes (Tong et al, 2018; Cheng et al, 2018). In cucumber, both opposite and positive trends between the expression levels of circRNAs and parental mRNAs have been reported (Zhu et al, 2019a).

In salt-sensitive cultivar 93-11, circRNA Chr1:30513415|30521330 was down-regulated and its parental gene LOC_Os01g53090, a pathogen-related protein, was up-regulated by $\mathrm{NaCl}$ stress (Table 2). Previous studies reported that LOC_Os01g53090 was up-regulated by both biotic and abiotic stresses such as Magnaporthe oryzae and aluminum stresses (Vijayan et al, 2013; Arbelaez et al, 2017). Another circRNA, Chr2:18305592|18305907, and its parental gene LOC_Os02g30714, a putative 11- $\beta$-hydroxysteroid dehydrogenase that has been proposed to take part in perception and transduction for many environmental stimuli (Wang et al, 2014), were up-regulated (Table 2, Table 3). In rice, LOC_Os02g30714, which was up-regulated by OsWRKY13, was an activator of rice in resistance to both bacterial and fungal pathogens, and was up-regulated during subsequent recovery after cold stress (Qiu et al, 2008; Yang et al, 2015). Chr4:18170122|18170775 was down-regulated and its parental gene LOC_Os04g30420, a zinc- 
binding dehydrogenase, was down-regulated. A previous study showed that LOC_Os04g30420 participated in the response to heat and drought stresses (Wilkins et al, 2016). These analyses reveal that many stress related genes in rice are the potential targets of circRNAs and could be differentially regulated by associated circRNAs in salt-sensitive cultivars.

Table 2. Differentially expressed circRNAs and corresponding parent genes in 93-11, and function annotation of parental genes.

\begin{tabular}{|c|c|c|c|c|}
\hline circRNA & $\begin{array}{l}\text { circRNA } \\
\text { regulated }\end{array}$ & Parent gene & $\begin{array}{l}\text { Parental } \\
\log 2(\mathrm{FC})\end{array}$ & Parental function \\
\hline Chr1:30513415|30521330 & down & LOC_Os01g53090 & 1.42 & $\begin{array}{l}\text { Pathogen-related } \\
\text { protein }\end{array}$ \\
\hline Chr1:38521288|38521427 & up & LOC_Os01g66330 & 1.98 & CLP protease \\
\hline Chr10:2483373|2483520 & up & LOC_Os10g05069 & 1.49 & a-mannosidase \\
\hline Chr11:4106581|4106802 & down & LOC_Os11g07940 & -1.77 & Centromere protein \\
\hline Chr12:14488613|14488960 & down & LOC_Os12g25200 & 2.92 & Chloride channel protein \\
\hline Chr2:16542324|16543258 & down & LOC_Os02g27950 & 1.35 & $\begin{array}{l}\text { Polyadenylate-binding } \\
\text { protein-interacting } \\
\text { protein }\end{array}$ \\
\hline Chr2:18305592|18305907 & up & LOC_Os02g30714 & 2.66 & $\begin{array}{l}\text { 11- } \beta \text {-hydroxysteroid } \\
\text { dehydrogenase }\end{array}$ \\
\hline Chr2:23868290|23871119 & up & LOC_Os02g39550 & -1.59 & $\begin{array}{l}\text { Calcium ion binding } \\
\text { protein }\end{array}$ \\
\hline Chr2:30459474|30459790 & up & LOC_Os02g49840 & 2.03 & $\begin{array}{l}\text { MADS-box transcription } \\
\text { factor }\end{array}$ \\
\hline Chr3:269113|269568 & down & LOC_Os03g01360 & -1.66 & Actin binding protein \\
\hline Chr3:35103079|35103219 & up & LOC_Os03g61920 & 2.12 & $\begin{array}{l}\text { Electron transfer } \\
\text { flavoprotein }\end{array}$ \\
\hline Chr3:8623603|8623803 & down & LOC_Os03g15630 & -1.59 & $\begin{array}{l}\text { Component of } \\
\text { membrane }\end{array}$ \\
\hline Chr4:18170122|18170775 & down & LOC_Os04g30420 & -2.09 & $\begin{array}{l}\text { Zinc-binding } \\
\text { dehydrogenase }\end{array}$ \\
\hline Chr6:3470974|3474679 & down & LOC_Os06g07250 & -2.07 & Jacalin-like lectin \\
\hline
\end{tabular}

Table 3 Differentially expressed circRNAs and corresponding parent genes in 9L-136, and function annotation of parental genes. 


\begin{tabular}{|c|c|c|c|c|}
\hline circRNA & $\begin{array}{l}\text { circRNA } \\
\text { regulated }\end{array}$ & Parent gene & $\begin{array}{l}\text { Parental } \\
\text { log2(FC) }\end{array}$ & Parental function \\
\hline Chr1:26607355|26607630 & up & LOC_Os01g46710 & 1.46 & $\begin{array}{l}\text { Translation initiation } \\
\text { factor }\end{array}$ \\
\hline Chr1:30512877|30520821 & down & LOC_Os01g53090 & 4.00 & $\begin{array}{l}\text { Pathogen-related } \\
\text { protein }\end{array}$ \\
\hline Chr2:18305592|18305907 & up & LOC_Os02g30714 & 4.50 & $\begin{array}{l}\text { 11- } \beta \text {-hydroxysteroid } \\
\text { dehydrogenase }\end{array}$ \\
\hline Chr2:23868553|23868970 & up & LOC_Os02g39550 & -1.65 & $\begin{array}{l}\text { Calcium ion binding } \\
\text { protein }\end{array}$ \\
\hline Chr2:4198743|4199218 & up & LOC_Os02g08010 & -2.07 & $\begin{array}{l}\mathrm{Ca}^{2+} \text {-transporting } \\
\text { ATPase }\end{array}$ \\
\hline Chr3:12838424|12838608 & down & LOC_Os03g22420 & 1.36 & AAA-ATPase FIGL-1 \\
\hline Chr3:30454536|30454982 & up & LOC_Os03g53110 & -1.43 & $\mathrm{Mg}^{2+}$ transporter \\
\hline Chr3:9760244|9760659 & down & LOC_Os03g17570 & 4.83 & $\begin{array}{l}\text { Signal transduction } \\
\text { regulator }\end{array}$ \\
\hline Chr4:18170122|18170775 & up & LOC_Os04g30420 & -1.43 & $\begin{array}{l}\text { Zinc-binding } \\
\text { dehydrogenase }\end{array}$ \\
\hline Chr4:30172735|30173954 & down & LOC_Os04g50970 & 4.00 & $\begin{array}{l}\text { Component of } \\
\text { membrane }\end{array}$ \\
\hline Chr5:21655167|21655378 & up & LOC_Os05g37060 & 4.79 & $\begin{array}{l}\text { Myb-like transcription } \\
\text { factor }\end{array}$ \\
\hline Chr8:18479857|18480021 & up & LOC_Os08g30060 & 1.31 & $\begin{array}{l}\text { Proton pump- } \\
\text { interactor }\end{array}$ \\
\hline Chr8:3226424|3226681 & up & LOC_Os08g05940 & 1.37 & RNA polymerase Rpb1 \\
\hline Chr9:4354645|4354999 & down & LOC_Os09g08390 & 1.47 & SEC14 cytosolic factor \\
\hline Chr10:20345844|20346873 & down & LOC_Os10g37980 & 2.10 & $\begin{array}{l}\text { Prephenate } \\
\text { dehydratase }\end{array}$ \\
\hline Chr11:23233410|23233648 & up & LOC_Os11g39020 & 2.13 & $A B C$ transporter \\
\hline Chr11:8467507|8467683 & down & LOC_Os11g15040 & -0.594708 & $\begin{array}{l}\text { Anthranilate 0- } \\
\text { methyltransferase }\end{array}$ \\
\hline Chr12:9356168|9356432 & down & LOC_Os12g16350 & 0.686881 & Enoyl-CoA hydratase \\
\hline
\end{tabular}

Similarly, in introgression line 93-11, LOC_Os01g53090, LOC_Os02g30714, LOC_Os02g39550, and LOC_OsO4g30420, the four parent genes of corresponding differentially regulated circRNAs, were also differentially expressed under $\mathrm{NaCl}$ treatment, implying the roles of these parent genes and circRNAs in 
salt stress response in both salt-susceptible and salt-tolerant rice cultivars (Table 3). Furthermore, many genes that function in ion transportation, such as $L O C \_0 s 02 g 08010\left(\mathrm{Ca}^{2+}\right.$-transporting ATPase, parental gene of Chr2:4198743|4199218), LOC_OsO3g53110 ( $\mathrm{Mg}^{2+}$ transporter, parental gene of Chr3:30454536|30454982), LOC_Os08g30060 (proton pump-interactor, parental gene of Chr8:18479857|18480021), LOC_Os02g39550 (a calcium ion binding protein, and parental gene of Chr2:23868290|23871119), and genes that function in hydrolase activity, such as LOC_Os10g37980 (prephenate dehydratase, parental gene of Chr10:20345844|20346873), and LOC_Os12g16350 (EnoylCoA hydratase, parental gene of Chr12:9356168|9356432), were differentially expressed (Table 3).

Furthermore, LOC_OSO5g31254 (calmodulin-related calcium sensor protein gene), a candidate gene for salt tolerance in rice (Wang et al, 2017c), was predicted to be the parent gene of circRNA

Chr6:2046190|2059120 in 93-11, and the parent gene of circRNA Chr10:18818949|18958248 in 9L-163. In Arabidopsis, the calmodulin-related calcium sensor protein gene has been reported to modulate stress responses. The functions of LOC_OSO5g31254 and Chr10:18818949|18958248 need to be further studied, since they will be valuable for determining salt stress mechanisms and conducting saltresistance breeding.

In summary, our study reveals the possible roles of rice circRNA in response to salt stress, which will expand our understanding of the characteristics of plant circRNAs and facilitate the determination of salt stress regulatory mechanisms in rice. Furthermore, the complicated relationships between the abundances of circRNAs and their parent genes need to be further explored through molecular biology approaches.

\section{Declarations}

\section{Funding}

This work was partially supported by the Open Project Program of Hubei Key Laboratory of Food Crop Germplasm and Genetic Improvement (2019lzjj03), the Open Project Program of Key Laboratory of Integrated Pest Management of Crops in Central China, Ministry of Agriculture/Hubei Key Laboratory of Crop Diseases, Insect Pests and Weeds Control (2019ZTSJJ8).

\section{Conflict of interest}

The authors declare that they have no conflict of interest.

\section{Availability of data and material}

All data generated or analyzed during this study are included in this published article and its supplementary information files. The datasets generated and analyzed during the current study are 
available from the corresponding author on reasonable request.

\section{Author contribution}

J.L. Y., Y.K. L., L. L., and J. Z. conceived the study, designed the experiments, and analyzed data. J.L. Y. wrote the first draft, S.Y. C. and B.T. W. finalized the manuscript. All the authors read the manuscript.

\section{Acknowledgments}

We thank LetPub (www.letpub.com) for its linguistic assistance during the preparation of this manuscript.

\section{References}

1. Arbelaez JD, Maron LG, Jobe TO, Piñeros MA, Famoso AN, Rebelo AR, Singh N, Ma Q, Fei Z, Kochian LV, Mccouch SR (2017) ALUMINUM RESISTANCE TRANSCRIPTION FACTOR 1 (ART1) contributes to natural variation in aluminum resistance in diverse genetic backgrounds of rice ( $O$. sativa). Plant Direct 1:e00014. https://doi.org/10.1002/pld3.14

2. Chen L, Zhang P, Fan Y, Lu Q, Li Q, Yan J, Muehlbauer GJ, Schnable PS, Dai M, Li L (2018) Circular RNAs mediated by transposons are associated with transcriptomic and phenotypic variation in maize. New Phytol 217:1292-1306. https://doi.org/10.1111/nph.14901

3. Cheng J, Zhang Y, Li Z, Wang T, Zhang X, Zheng B (2018) A lariat-derived circular RNA is required for plant development in Arabidopsis. Sci China Life Sci 61:204-213. https://doi.org/10.1007/s11427017-9182-3

4. Cho KM, Nguyen HT, Kim SY, Shin JS, Cho DH, Hong SB, Shin JS, Ok SH (2016) CML10, a variant of calmodulin, modulates ascorbic acid synthesis. New Phytol 209:664-678. https://doi.org/10.1111/nph.13612

5. Chu Q, Zhang X, Zhu X, Liu C, Mao L, Ye C, Zhu Q, Fan L (2017) PlantcircBase: A database for plant circular RNAs. Mol Plant 10:1126-1128. https://doi.org/10.1016/j.molp.2017.03.003

6. Chu Q, Bai P, Zhu X, Zhang X, Mao L, Zhu QH, Fan L, Ye CY (2020) Characteristics of plant circular RNAs. Brief bioinform 21:135-143. https://doi.org/10.1093/bib/bby111

7. Conn VM, Hugouvieux V, Nayak A, Conos SA, Capovilla G, Cildir G, Jourdain A, Tergaonkar V, Schmid M, Zubieta C, Conn SJ (2017) A circRNA from SEPALLATA3 regulates splicing of its cognate mRNA through R-loop formation. Nat Plants 3:17053. https://doi.org/10.1038/nplants.2017.53

8. Fang Z, Jiang W, He Y, Ma D, Liu Y, Wang S, Zhang Y, Yin J (2020) Genome-wide identification, structure characterization, and expression profiling of Dof transcription factor gene family in wheat (Triticum aestivum L.). Agronomy 10:294. https://doi.org/10.3390/agronomy10020294 
9. Gao Y, Zhang J, Zhao F (2017) Circular RNA identification based on multiple seed matching. Brief Bioinform 19:803-810. https://doi.org/10.1093/bib/bbx014

10. Gong HJ, Randall DP, Flowers TJ (2006) Silicon deposition in the root reduces sodium uptake in rice (Oryza sativa L.) seedlings by reducing bypass flow. Plant Cell Environ 29:1970-1979. https://doi.org/10.1111/j.1365-3040.2006.01572.x

11. He X, Guo S, Wang Y, Wang L, Shu S, Sun J (2020) Systematic identification and analysis of heatstress-responsive IncRNAs, circRNAs and miRNAs with associated co-expression and ceRNA networks in cucumber (Cucumis sativus L.). Physiol Plantarum 168:736-754. https://doi.org/10.1111/ppl.12997

12. Huang X, Feng J, Wang R, Zhang H, Huang J (2019) Comparative analysis of microRNAs and their targets in the roots of two cultivars with contrasting salt tolerance in rice (Oryza sativa L.). Plant Growth Regul 87:139-148. https://doi.org/10.1007/s10725-018-0459-4

13. Li HM, Ma XL, Li HG (2019) Intriguing circles: Conflicts and controversies in circular RNA research. Wires RNA 10:e1538. https://doi.org/10.1002/wrna.1538

14. Li X, Liu C, Xue W, Zhang Y, Jiang S, Yin Q, Wei J, Yao R, Yang L, Chen L (2017) Coordinated circRNA biogenesis and function with NF90/NF110 in viral infection. Mol Cell 67:214-227. https://doi.org/10.1016/j.molcel.2017.05.023

15. Liu J, Liu T, Wang X, He A (2017) Circles reshaping the RNA world: from waste to treasure. Mol Cancer 16:58. https://doi.org/10.1186/s12943-017-0630-y

16. Liu T, Zhang L, Chen G, Shi T (2017) Identifying and characterizing the circular RNAs during the lifespan of Arabidopsis leaves. Front Plant Sci 8:1278. https://doi.org/10.3389/fpls.2017.01278

17. Lu T, Cui L, Zhou Y, Zhu C, Fan D, Gong H, Zhao Q, Zhou C, Zhao Y, Lu D, Luo J, Wang Y, Tian Q, Feng Q, Huang T, Han B (2015) Transcriptome-wide investigation of circular RNAs in rice. RNA 21:20762087. https://doi.org/10.1261/rna.052282.115

18. Mangrauthia SK, Bhogireddy S, Agarwal S, Prasanth VV, Voleti SR, Neelamraju S, Subrahmanyam D (2017) Genome-wide changes in microRNA expression during short and prolonged heat stress and recovery in contrasting rice cultivars. J Exp Bot 68:2399-2412

19. Meng X, Li X, Zhang P, Wang J, Zhou Y, Chen M (2016) Circular RNA: an emerging key player in RNA world. Brief Bioinform 18:547-557. https://doi.org/10.1093/bib/bbw045

20. Pan T, Sun X, Liu Y, Li H, Deng G, Lin H, Wang S (2018) Heat stress alters genome-wide profiles of circular RNAs in Arabidopsis. Plant Mol Biol 96:217-229. https://doi.org/10.1007/s11103-017-06847

21. Qin Z, Chen J, Jin L, Duns GJ, Ouyang P (2015) Differential expression of miRNAs under salt stress in Spartina alterniflora leaf tissues. J Nanosc Nanotechno 15:1554-1561. https://doi.org/10.1166/jnn.2015.9004

22. Qiu D, Xiao J, Xie W, Liu H, Li X, Xiong L, Wang S (2008) Rice gene network inferred from expression profiling of plants overexpressing OsWRKY13, a positive regulator of disease resistance. Mol Plant 1:538-551. https://doi.org/10.1093/mplant/ssn012 
23. Tan J, Zhou Z, Niu Y, Sun X, Deng Z (2017) Identification and functional characterization of tomato circRNAs derived from genes involved in fruit pigment accumulation. Sci Rep 7:8594. https://doi.org/10.1038/s41598-017-08806-0

24. Tong W, Yu J, Hou Y, Li F, Zhou Q, Wei C, Bennetzen JL (2018) Circular RNA architecture and differentiation during leaf bud to young leaf development in tea (Camellia sinensis). Planta 248:1417-1429. https://doi.org/10.1007/s00425-018-2983-x

25. Vijayan J, Jain S, Jain N, Devanna B, Rathour R, Variar M, Prashanthi S, Singh A, Singh U, Singh N, Sharma T (2013) Identification of differentially expressed genes in rice during its early phases of interaction with Magnaporthe oryzae. Indian J Genet 73:233-243. https://doi.org/10.5958/j.09756906.73.3.035

26. Wang K, Wang C, Guo B, Song K, Shi C, Jiang X, Wang K, Tan Y, Wang L, Wang L, Li J, Li Y, Cai Y, Zhao H, Sun X (2019) CropCircDB: a comprehensive circular RNA resource for crops in response to abiotic stress. Database 2019:baz053. https://doi.org/10.1093/database/baz053

27. Wang L, Chu H, Li Z, Wang J, Li J, Qiao Y, Fu Y, Mou T, Chen C, Xu J (2014) Origin and development of the root cap in rice. Plant Physiol 166:603-613. https://doi.org/10.1104/pp.114.240929

28. Wang S, Cao M, Ma X, Chen W, Zhao J, Sun C, Tan L, Liu F (2017c) Integrated RNA sequencing and QTL mapping to identify candidate genes from Oryza rufipogon associated with salt tolerance at the seedling stage. Front Plant Sci 8:1427. https://doi.org/10.3389/fpls.2017.01427

29. Wang Y, Yang M, Wei S, Qin F, Zhao H, Suo B (2017a) Identification of circular RNAs and their targets in leaves of Triticum aestivum L. under dehydration stress. Front Plant Sci 7:2024. https://doi.org/10.3389/fpls.2016.02024

30. Wang Z, Liu Y, Li D, Li L, Zhang Q (2017b) Identification of circular RNAs in kiwifruit and their speciesspecific response to bacterial canker pathogen invasion. Front Plant Sci 8:413. https://doi.org/10.3389/fpls.2017.00413

31. Wang YG, An M, Zhou SF, She YH, Li WC, Fu FL (2014) Expression profile of maize microRNAs corresponding to their target genes under drought stress. Biochem Genet 52:474-493. https://doi.org/10.1007/s10528-014-9661-x

32. Wilkins $O$, Hafemeister C, Plessis A, Holloway-Phillips M-M, Pham GM, Nicotra AB, Gregorio GB, Jagadish SVK, Septiningsih EM, Bonneau R, Purugganan M (2016) EGRINs (Environmental Gene Regulatory Influence Networks) in rice that function in the response to water deficit, high temperature, and agricultural environments. Plant Cell 28:2365-2384. https://doi.org/10.1105/tpc.16.00158

33. Xu X, Bai H, Liu C, Chen E, Chen Q, Zhuang J, Shen B (2014) Genome-wide analysis of microRNAs and their target genes related to leaf senescence of rice. PLoS ONE 9:e114313. https://doi.org/10.1371/journal.pone.0114313

34. Yang YW, Chen HC, Jen WF, Liu LY, Chang MC (2015) Comparative transcriptome analysis of shoots and roots of TNG67 and TCN1 rice seedlings under cold stress and following subsequent recovery: Insights into metabolic pathways, phytohormones, and transcription factors. PLoS ONE 10:e0131391. https://doi.org/10.1371/journal.pone.0131391

Page $15 / 23$ 
35. Ye C, Zhang X, Chu Q, Liu C, Yu Y, Jiang W, Zhu Q, Fan L, Guo L (2016) Full-length sequence assembly reveals circular RNAs with diverse non-GT/AG splicing signals in rice. RNA Biol 14:10551063. https://doi.org/10.1080/15476286.2016.1245268

36. Ye CY, Chen L, Liu C, Zhu QH, Fan L (2015) Widespread noncoding circular RNAs in plants. New Phytol 208:88-95. https://doi.org/10.1111/nph.13585

37. Yin J, Liu M, Ma D, Wu J, Li S, Zhu Y, Han B (2018) Identification of circular RNAs and their targets during tomato fruit ripening. Postharvest Biol Tec 136:90-98. https://doi.org/10.1016/j.postharvbio.2017.10.013

38. You X, Vlatkovic I, Babic A, Will T, Epstein I, Tushev G, Akbalik G, Wang M, Glock C, Quedenau C, Wang X, Hou J, Liu H, Sun W, Sambandan S, Chen T, Schuman EM, Chen W (2015) Neural circular RNAs are derived from synaptic genes and regulated by development and plasticity. Nat Neurosci 18:603-610. https://doi.org/10.1038/nn.3975

39. Zare S, Nazarian-Firouzabadi F, Ismaili A, Pakniyat $H$ (2019) Identification of miRNAs and evaluation of candidate genes expression profile associated with drought stress in barley. Plant Gene 20:100205. https://doi.org/10.1016/j.plgene.2019.100205

40. Zhang M, Huang N, Yang X, Luo J, Yan S, Xiao F, Chen W, Gao X, Zhao K, Zhou H, Li Z, Ming L, Xie B, Zhang N (2018) A novel protein encoded by the circular form of the SHPRH gene suppresses glioma tumorigenesis. Oncogene 37:1805-1814. https://doi.org/10.1038/s41388-017-0019-9

41. Zhao W, Cheng Y, Zhang C, You Q, Shen X, Guo W, Jiao Y (2017) Genome-wide identification and characterization of circular RNAs by high throughput sequencing in soybean. Sci Rep 7:5636

42. Zhao W, Chu S, Jiao Y (2019) Present scenario of circular RNAs (circRNAs) in plants. Front Plant Sci 10:379. https://doi.org/10.1038/s41598-017-05922-9

43. Zhou R, Zhu Y, Zhao J, Fang Z, Wang S, Yin J, Chu Z, Ma D (2018) Transcriptome-wide identification and characterization of potato circular RNAs in response to Pectobacterium carotovorum subspecies brasiliense infection. Int J Mol Sci 19:71. https://doi.org/10.3390/ijms19010071

44. Zhu YX, Jia JH, Yang L, Xia YC, Zhang HL, Jia JB, Zhou R, Nie PY, Yin JL, Ma DF, Liu LC (2019a) Identification of cucumber circular RNAs responsive to salt stress. BMC Plant Biol 19:164. https://doi.org/10.1186/s12870-019-1712-3

45. Zhu Y, Gong H, Yin J (2019b) Role of silicon in mediating salt tolerance in plants: A review. Plants 8:147. https://doi.org/10.3390/plants8060147

46. Zhu Y, Yang L, Liu N, Yang J, Zhou X, Xia Y, He Y, He Y, Gong H, Ma D, Yin J (2019c) Genome-wide identification, structure characterization, and expression pattern profiling of aquaporin gene family in cucumber. BMC Plant Biol 19:345. https://doi.org/10.1186/s12870-019-1953-1

\section{Figures}



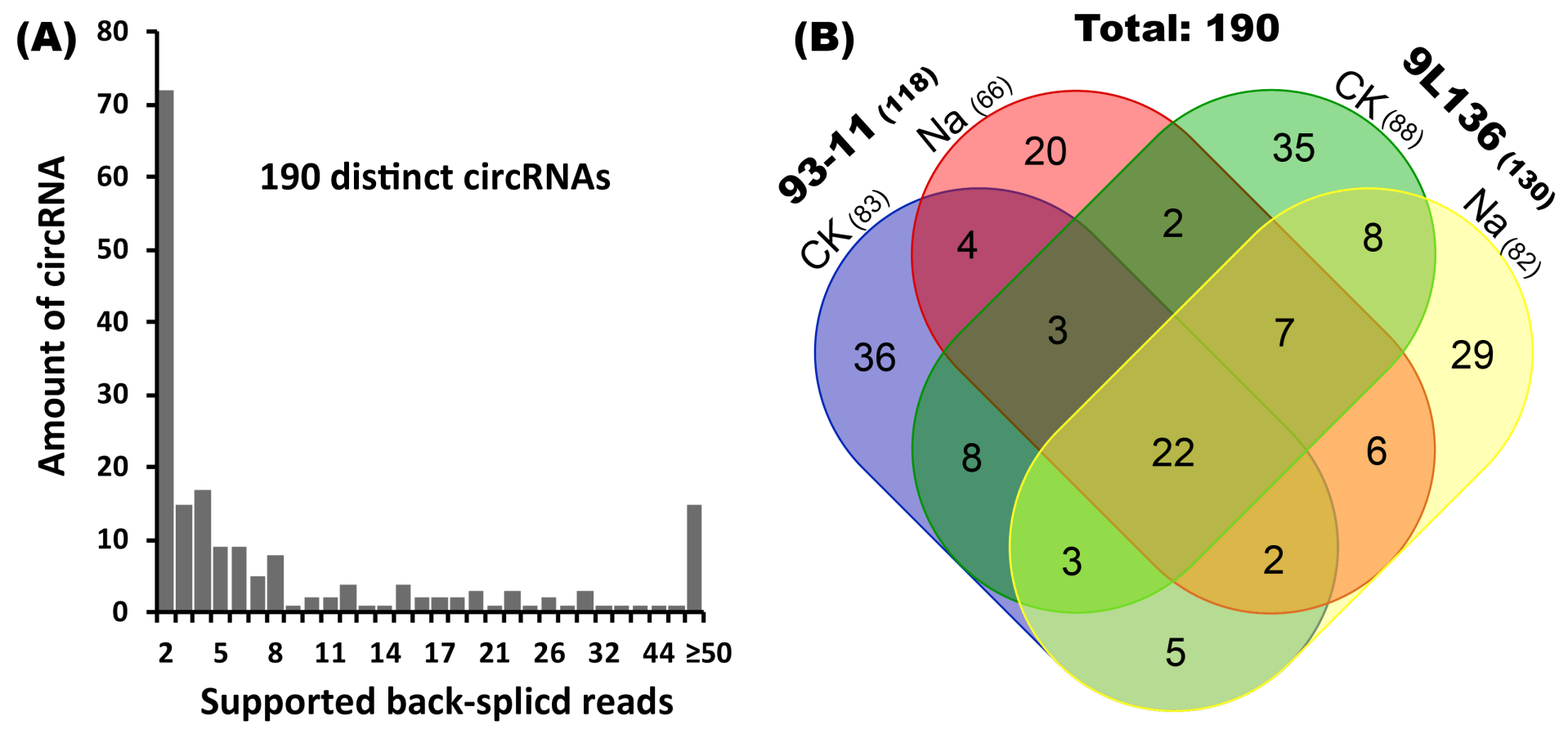

Figure 1

The distribution of circRNAs in recipient $93-11$ and introgression line 9 L136 between control (CK) and salt stress $(\mathrm{NaCl})$ treatments. $(A)$ Detected number of circRNAs and their supporting back-splicing junction reads. (B) Venn diagram showing the number and distribution of circRNA in 93-11 and 9L136 under CK and $\mathrm{NaCl}$ conditions. 


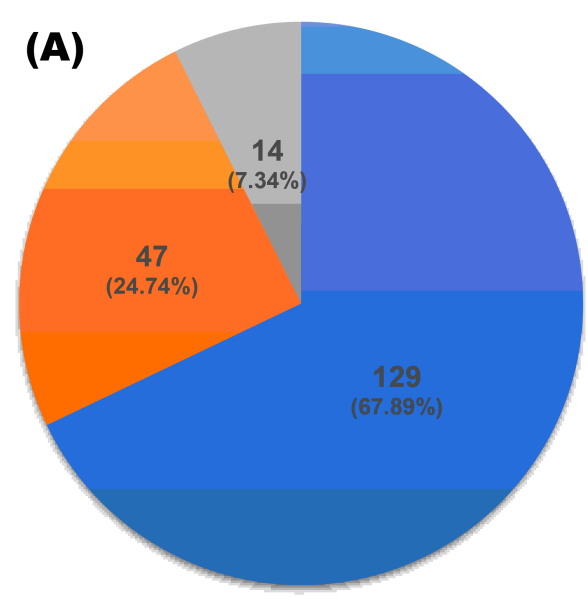

- intergenic_region =exon =intron

(D)

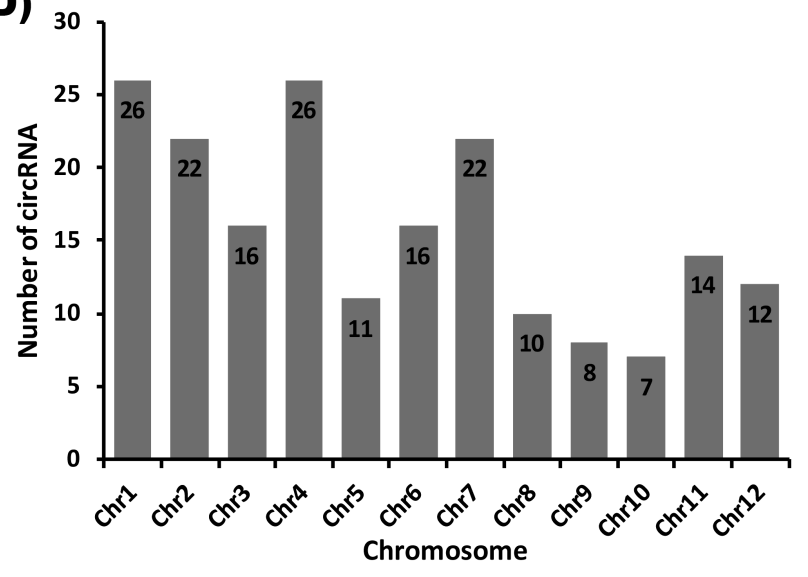

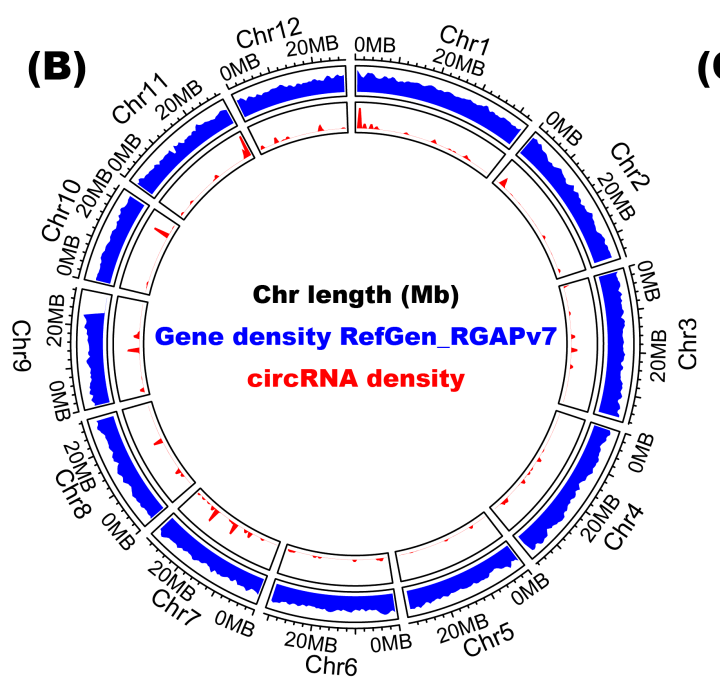
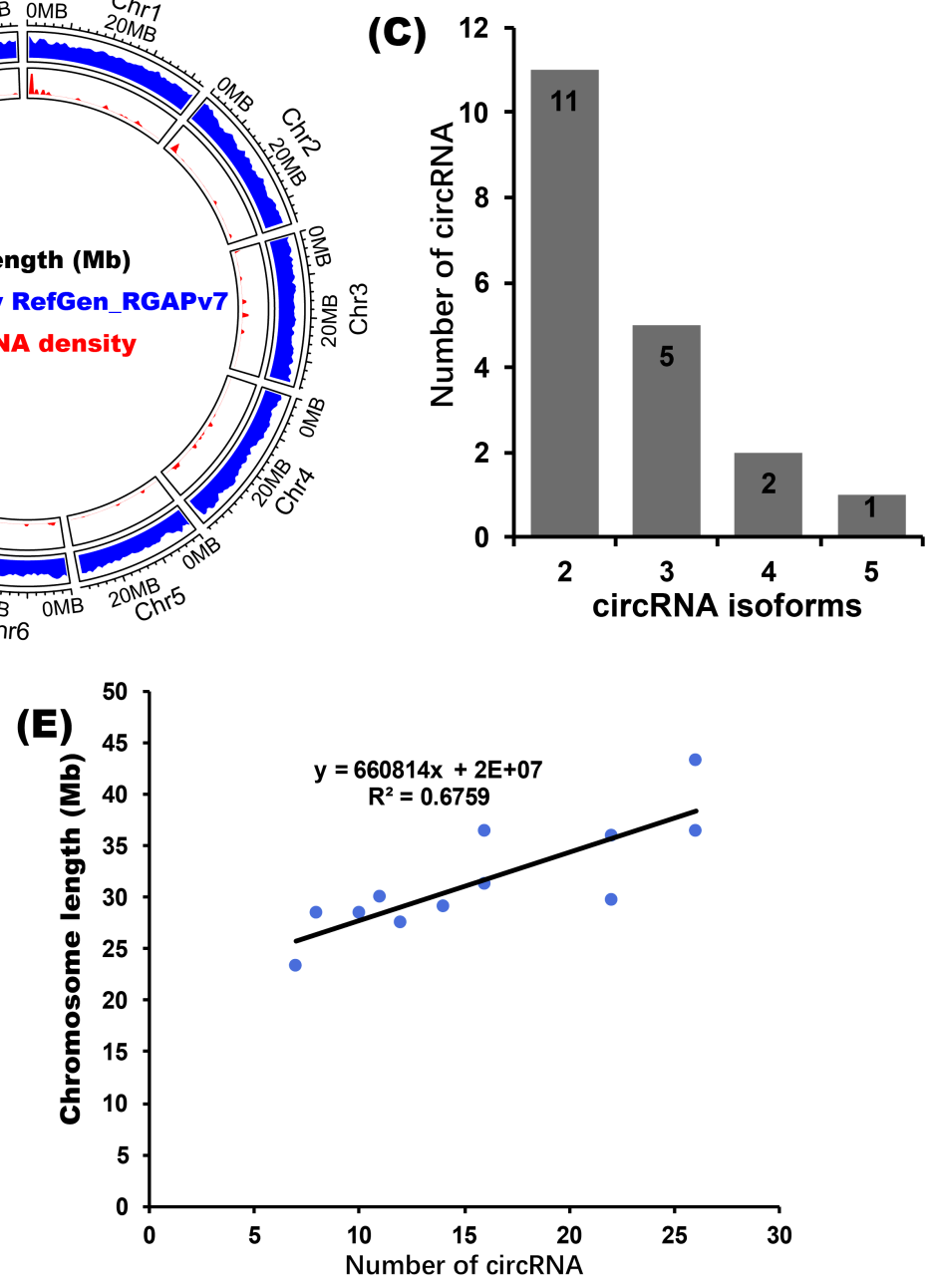

\section{Figure 2}

Characterization of rice circRNAs. (A) Pie chart representing the number and percentage of circRNAs generated from intergenic, exon, and intron regions. (B) Circos showing the distribution and density of reference mRNAs and identified circRNAs in each chromosome. (C) Statistic of alternative back-splicing circularization events. (D) Histogram showing the number of circRNAs detected in each chromosome. (E) Correlation analysis between chromosome length and the number of circRNA. 

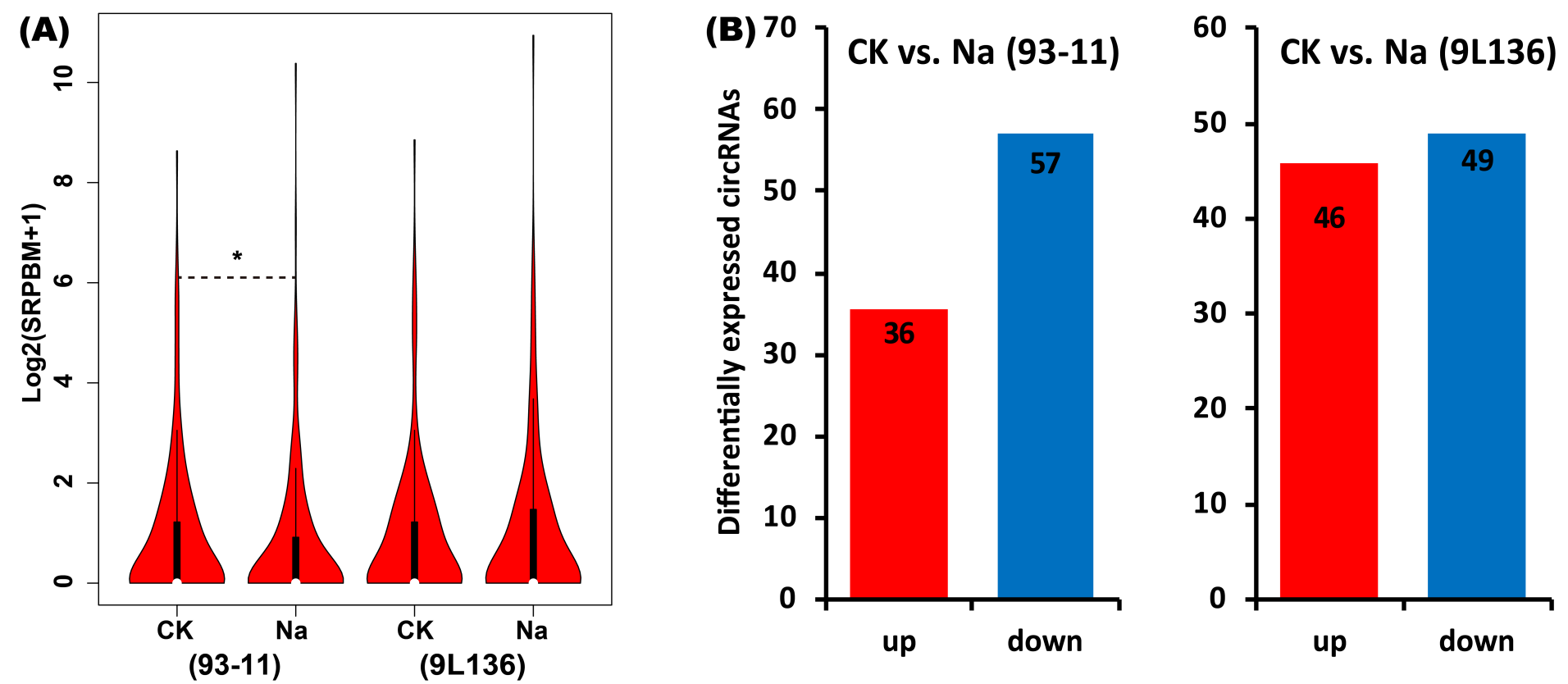

Figure 3

Differentially expressed circRNAs in response to salt stress. (A) Violin plot of relative abundance of circRNAs in salt stress tissues compared with the corresponding control tissues. Data are represented as the $\log 2($ SRPBM +1$)$. The white dot represents the median. ${ }^{*} p<0.05$, Wilcoxon rank-sum test. (B) Number of differentially expressed circRNAs. 93-11 and 9L136, two rice cultivars; vs., versus. 


\section{(A) 9 L136}

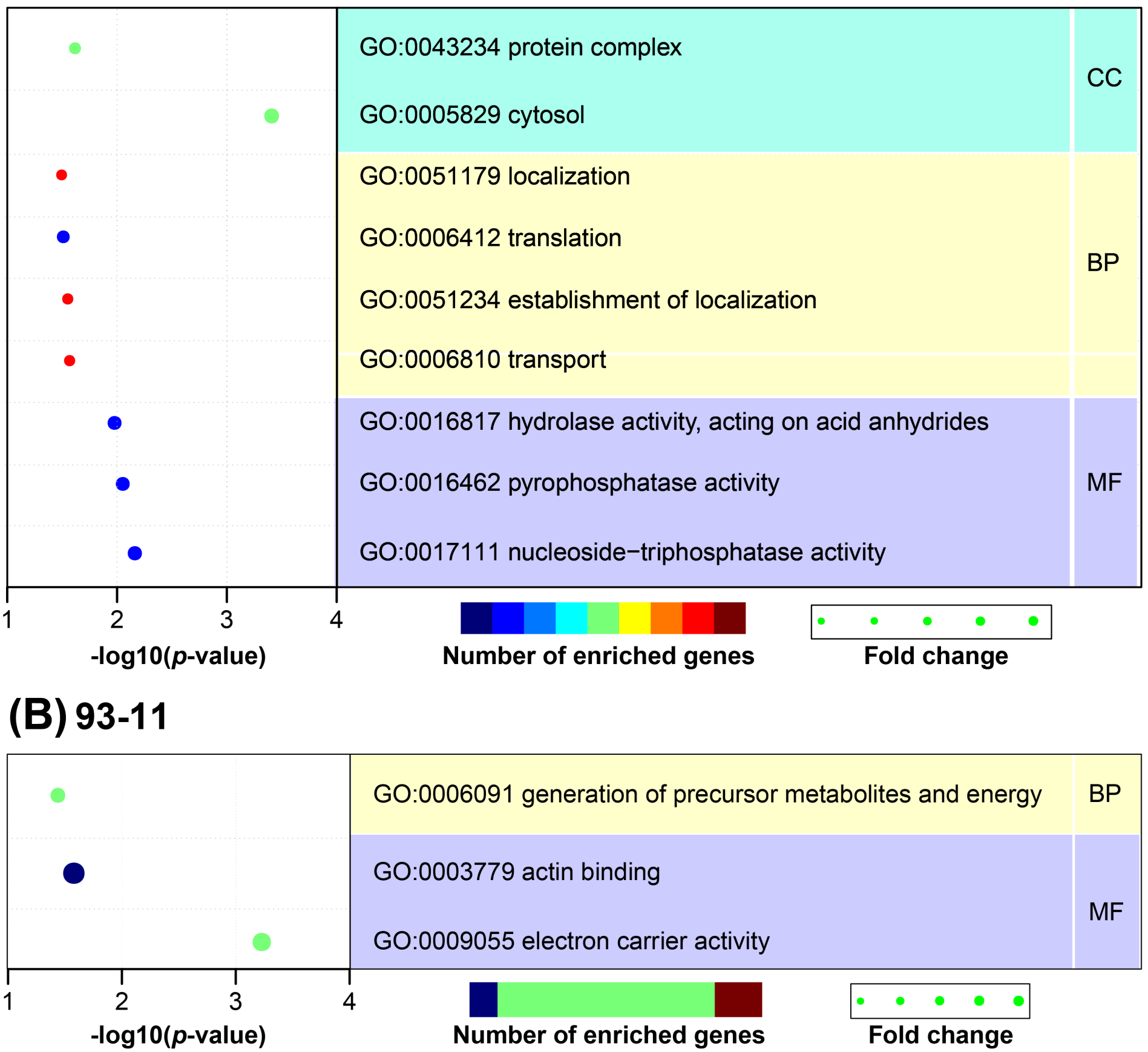

Figure 4

GO enrichment analysis of differentially expressed circRNAs in two rice cultivars. (A) 9L136 and (B) 9311. GO, Gene Ontology; CC, cellular component; MF, molecular function; BP, biological process. 


\section{(A) 9L-136}

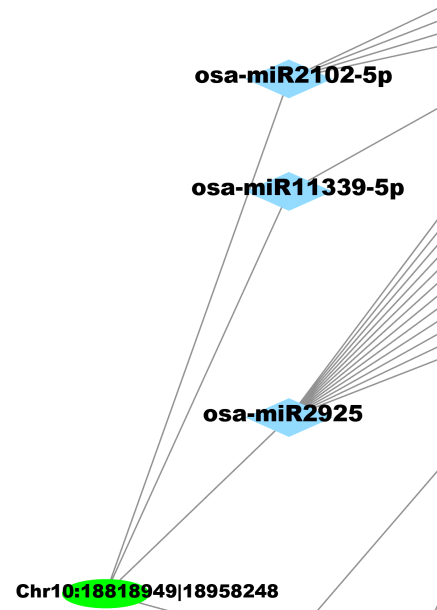

osa-miR395o

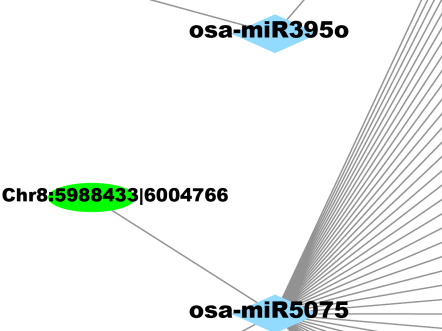

Chr8:5954285|6001870

Chr12:5852299|5902166

osa-miR390-3p
LOC_OS12g06850 mediator of RNA polymerase II LOC_Os05g25390 Wall-associated receptor kinase LOC Os06912290 glutathione S-transferase LOC Os03g46570 BOl-related E3 ubiquitin-protein LOC_Os08g03560 Protein CHUP1

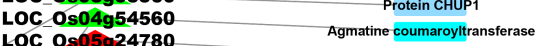
LOC_Os05924780 $\quad$ Agmatine coumaroyltransferase LOC Os $01943320 \quad$ calcium-binding protein YLt nuclear protein Lec 0502946990 Protein QUIRKY AAA-ATPase

$\begin{array}{lc}\text { LOC OS } 09928200 & \text { Histone } \mathrm{H} 1 \\ \text { LOC Os10g39190 } & \text { Heat stress transcription factor } \mathrm{B}-4\end{array}$

B3 domain-containing protein

LOC Os10g35440 Protein POLLENLESS 3-LIKE 2

LOC Os03953230 Protein Rf1

LOC_0s02g27060 Pre-mRNA-splicing factor

LOC Os06g11240 ATP-sulfurylase

LOC_0s02g1778

LOC_0s12g24020

LOC Os09g25600

LOC Os05g49940

LOC 0 s02g56250

LOC 050890439

Oc 0 s07g2889

OC Os08g3944

OC 0 s02g5032

$0 c 0$ 0293065

oc Os 0390298

Loc Os $05 \mathrm{~g} 35460$

OC Os $03 \mathrm{~g} 12120$

LOC 0 s12g07530

Loc 0 s02g54650

Loc Os06g14050

OC 0 03908740

OC Os08g1502

CC 0 s03g60850

OC Oso3g 25760

LOC Os09g23740

LOC Os11g11340

LOC_Os11g11340

LOC Os02g39930

LOC Os11g47650

LOC_Os09g25040

OC 0.02941840

LCC Os01915000

LOC Os01913210

LOC Os10g23050

LC Os10g 3696

LOC Os06g 19070

LOC Os08g26820

LOC Os03g14880

LOC_0s09g27330

LOC Os06g10020

LOC Os02914490

LOC_Os02g57810

LOC_Os06g22340

LOC Os08g05620

LOC Os05g45040

Loc Os10g04840

oc 0.9893906

Loc Os03g25280

LOC Os03g25280

LOC_Os03939850

LOC Os06g0383

LOC $0 s 0290982$

LOC_Os05g42180

LOC_0s11g02070

LOC Os05g06780

LOC Os10g01100

LOC Os029 39570

LOC O 01903510

LOC Os05g04690

LOC Os06g13180

LOC Os01924880

Loc Os03g6058

LOC Os06g30090

Loc Os0494997

LC Os01g0658

LOC Os08g34360

LOC_Os03g21160

LOC_Os02g10480

LOC Os06g06802

Transcription

NADH:flavin oxidoreductase

Ent-copalyI diphosphate synthase

Thiosulfate sulfurtransferase

WRINKLED1-like protein

forkhead box protein L2

GATA transcription factor

Transcription factor ILR3

$33 \mathrm{kDa}$ ribonucleoprotein

Transcription factor bHLH111

Polyadenylate-binding protein

Microtubule-associated protein

Inorganic ion transport and
metabolism

Serine/threonine-protein kinase

patellin-3

NAM protein
Gene silencing associated protein

Diacylglycerol kinase

MACPE domain-containing protein

Transmembrane protein 131-like

Transcription factor MYB3

NRT1/PTR protein IQM2

Auxin efflux carrier

Phosphatidylinositol-4-phosphate
5-Kinase

Calmodulin-interacting protein

Temperature-induced lipocalin

Protein ALP1-like

Activator of Hsp90 ATPase

HLH transcription factor PTF1-like
protein Chr11:28076662/28116956

Senescence regulator

Lipase

Salt stress root protein RS 1

Transcription factor bHLH9

Membrane component

Myb-like transcription factor

Cytochrome P450

F-box protein

Peroxidase

Glutathione S-transferase

E3 ubiquitin-protein ligase RING

Transcription factor bHLH112

TB/POZ domain-containing protei lipid-transfer protein G-type lectin S-receptor-like
serinethroeonine-protetein kinase Amino acid transport and
metabolism Cytoskeleton stress prot Chitinase 10 Metalloendoproteinas

E3 ubiquitin-protein ligase RFI2 Actin-depolymerizing factor transcription factor SPATULA

E3 ubiquitin-protein ligase PUB23 membrane biogenesis AP2/EREBP transcription factor F-box protein AFR Zinc finger protein ADP-ribosylation factor Reverse transcriptase
(B) 93-11

osa-miR159f

Chr1:1170419|1348715 osa-miR159c

Chr6:2046190|2059120

osa-miR2925

osa-miR6249b

Chr1:35587727|35598212

Chr8:5954285|6001870

Chr1:4755137|4796461 osa-miR390-3p

OC 0506940330 LOC 0\$04g40590 LOC OS09928690

LOC 0s03g16760

LOC Os01g51320

LOC Os08g41800

LOC Os09g27500

LOC_Os $04 \mathrm{~g} 54560$

LOC Os $10 \mathrm{~g} 35240$

LOC Os01g 43320

LOC $0 \leq 08 \mathrm{~g} 08080$

Loc Os $10 \mathrm{~g} 39190$

LCC 0s05g 08560

LOC Os 03944890

LOC 0504g20590

LOC Os10g35440

LOC OS03a58470

LOC Os09g36740

LOC Os09g28200

LOC Os02g4699

LOC 0s06g5005

LOC OS03g44740

Loc Os11g44940

cc 0294510

LOC OS07g05940

LOC 0.11947650

LOC Os10g18150

LOC Os 02941840

LOC OSO1g1500

LOC Os $03 \mathrm{~g} 1212$

LOC OS12g24020

LOC Os 09g2 25040

LOC Os $03 \mathrm{~g} 02980$

LOC Os 08904390

Loc Os 02950320

LOC 0s07g28890

LOC 0 02939930

LOC Os07g40870

LC Os03g0314

LOC Os02g54650

Loc 0s03g60850

LOC 0s $03 \mathrm{~g} 25760$

Loc 0s $05 \mathrm{g02490}$

LOC Os $12 \mathrm{~g} 07530$

osa-miR5075 LOC Os05g35460

LOC Os09g23740

LOC 0502930650

LOC Os03g39720

LOC $0503 \mathrm{~g} 07180$

LOC 0502917780

LOC Os09g25600

Loc OS 10 g 23050

LOC OS08915020

LC 0 06949030

Loc Os01945550

LOC Os01g45550

LOC Os06g14050

LOC Os02g56250

LOC Os06g10450

LOC Os06g11240

LOC OS03g08740

LOC OSO9g27330

LOC Os03g14880

LOC Os08g26820

LOC Os02g58170

LOC Os03g30519

Chr4:32061777|32087319 osa-miR1850.3 LOC Os12g06610

MiRNA

mRNA

CircRNA

Transcription

Enzyme

Transport

Component

Figure 5

Possible regulatory networks involving differentially expressed circRNAs and their target genes during rice responding to salt stress in (A) 9L-136 and (B) 93-11. Circle, diamond, and triangle represent circular RNA, miRNA, and mRNA. Red and green nodes represent up- and down-regulated different expression genes. Rectangle nodes represent the corresponding function annotation of mRNAs. The pink, magenta, cyan, and coral rectangle nodes represent categories of transcription, transport, enzyme, and component, 
respectively. Detailed information, including binding sites, relationship among circRNAs, miRNAs, and mRNAs, and annotation of mRNAs were listed in Table S4, S5.

(A)

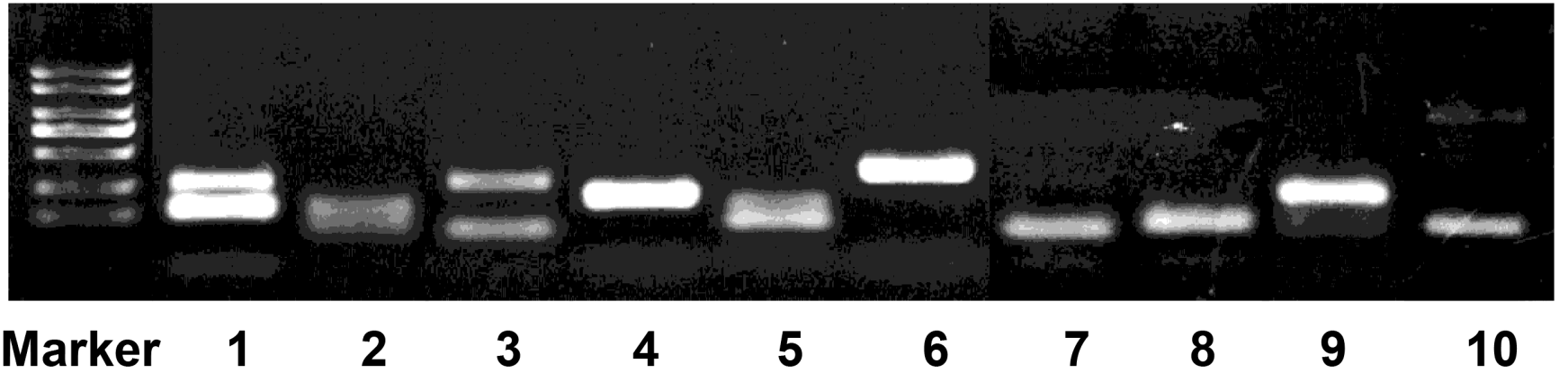

(B)

Model

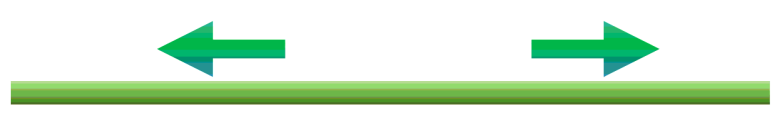

Divergent primer

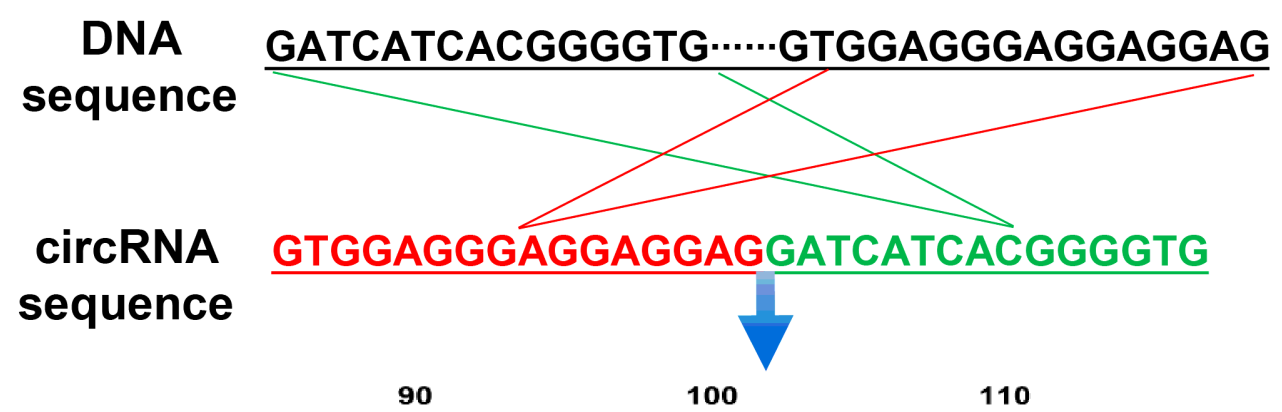

GTGGAGGGAGGAGGAGGATCATCACG G GGTGG

\section{Sanger sequencing}
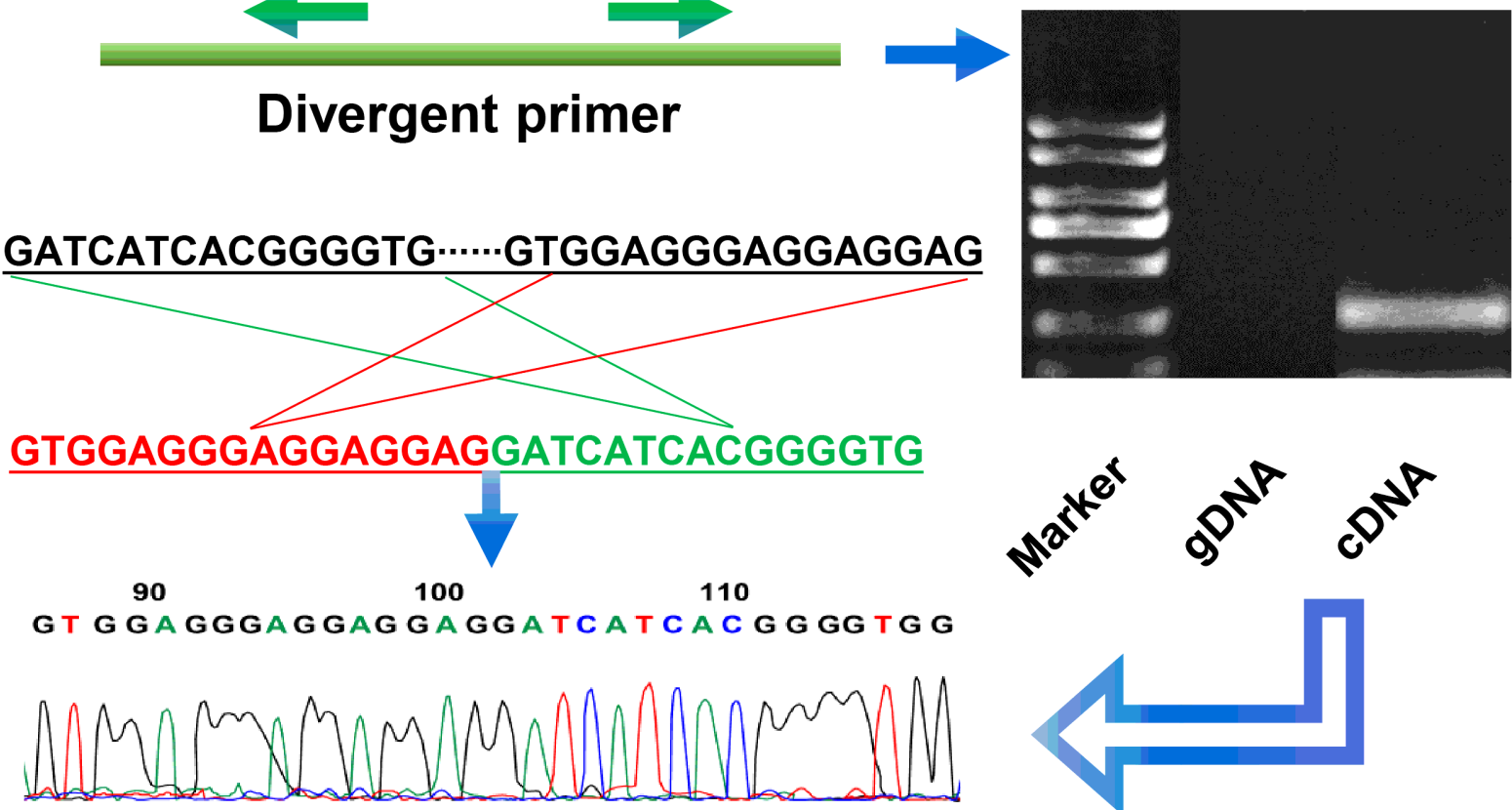

Chr10:20345844|20346873

Figure 6

Validation of rice circRNA through PCR and Sanger sequencing. (A) M, marker; 1 to 10 , ten circRNA validation using Sanger sequencing. (Detailed information, including the corresponding ID of 1 to 10 circRNAs and their divergent primers, can be found in Supplementary table 6). (B) using Chr10:20345844|20346873 as an example. Upper left panel (Model), a model used to show the divergent primers for circRNAs backsplicing sites amplification. Upper right panel, an example showing that amplification of divergent primers can be amplified from cDNA but not from genomic DNA. Two middle panels, respectively, black rectangle (DNA sequence) representing downstream and upstream sequences in the genome, and red and green rectangles (circRNA sequence) representing back-splicing circRNA sequence. Lower panel, a Sanger sequencing example of cucumber circRNA Chr10:20345844|20346873. 


\section{Supplementary Files}

This is a list of supplementary files associated with this preprint. Click to download.

- SupplementarytableLegends.docx

- Supplementalfigure1.docx

- Supplementarytables.xlsx 